\title{
ALGEBRAIC QUOTIENTS OF BERGMAN DOMAINS
}

\author{
GONZALO G. RIERA
}

ABSTRACT. A discrete group of automorphisms of a domain in $\mathbf{C}^{n}$ is constructed so that the quotient is algebraic.

A Bergman domain in $\mathbf{C}^{n}$ is a set $D=\left\{\left(z_{1}, \ldots, z_{n}\right) / z_{1} \in U, z_{i+1} \in\right.$ $\left.B_{i+1}\left(z_{1}, \ldots, z_{i}\right), i=1, \ldots, n-1\right\}$ where $B_{i+1}$ is a Jordan domain in $\mathbf{C}$ whose boundary admits a parametric representation

$$
\zeta_{i+1}=W\left(z_{1}, \ldots, z_{i} ; x\right), \quad x \in \mathbf{R} .
$$

For fixed $x W$ is a holomorphic function of $\left(z_{1}, \ldots, z_{i}\right) \in D_{i}$, the projection of $D$ onto the first $i$ coordinates.

Given an arbitrary quasi-projective algebraic variety $A_{0}$ there is a Zariski open subset $A_{1} \subset A_{0}$ such that the universal covering of $A_{1}$ is a Bergman domain (cf. Griffiths [2], Bers [1]). In this note we prove a partial converse to the theorem above, namely that a discrete group of analytic automorphisms of $D$, obtained as an extension of certain Fuchsian groups of finite type, gives as quotient an algebraic variety.

Let $\mu$ be a measurable function in the upper half plane $U$ with $\|\mu\|_{\infty}<1$. We denote by $W^{\mu}$ the unique homeomorphic solution of the Beltrami equation

$$
\partial W^{\mu} / \partial \bar{z}=\mu \partial W^{\mu} / \partial z
$$

fixing 0,1 and $\infty$, where $\mu$ is extended by 0 to the lower half plane. The domains considered here are to satisfy the following additional property:

For any point $i \in D_{i}$ there is a neighborhood $i \in V \subset D_{i}$ and a holomorphic function $\alpha_{i}: V \rightarrow L_{1}^{\infty}(U)$ such that $B_{i+1}=W^{\alpha_{i}(z)}(U)$ for all $z \in V$.

Consider an ascending chain of groups

$$
\{1\}=G_{0} \triangleright G_{1} \triangleright \cdots \triangleright G_{n}=G
$$

where $G_{i+1}$ is a split extension $G_{i}$ by a Fuchsian group $H_{i}(0 \leqslant i \leqslant n-1)$. Assume further that there is an analytic function $f_{i}: D_{i} \rightarrow T\left(H_{i}\right)$ into the Teichmüller space of $H_{i}$, "coinciding" with $\alpha_{i}$ in each neighborhood $V$, such that $f_{i}$ maps $G_{i}$ into a subgroup of the Teichmüller modular group $\operatorname{Mod}\left(H_{i}\right)$.

It is then possible (cf. Riera [4]) to define by recurrence an action of $G_{i+1}$ on $D_{i+1}$ by means of the formula 


$$
(g, h)(z, \zeta)=\left(g(z), W^{f_{i}(g(z))} \circ h \circ \delta_{i}(g) \circ\left(W^{f_{i}(z)}\right)^{-1}(\zeta)\right)
$$

for all $z \in D_{i}, \zeta \in B_{i+1}, g \in G_{i}, h \in H_{i}$, where $\delta_{i}$ is an appropriate homomorphism from $G_{i}$ into a $\operatorname{group} \bmod \left(H_{i}\right)$.

THEOREM. Let $G$ be a group of analytic automorphisms of a Bergman domain $D$, obtained by a chain of split extensions of Fuchsian groups $H_{i}$ of finite type $\left(P_{i}, K_{i}\right), 0 \leqslant i \leqslant n-1$. If $K_{i}=0$ for $0 \leqslant i \leqslant n-2$, then $D / G$ is a quasiprojective algebraic variety.

Proof. If $\phi_{i}, \psi_{i}$ denote fundamental regions for $G_{i}$ and $H_{i}$ respectively, then a fundamental region for $G_{i+1}$ on $D_{i+1}$ is the set

$$
\phi_{i+1}=\left\{(z, \zeta) / z \in \phi_{i}, \zeta \in W^{f_{i}(z)}\left(\psi_{i}\right)\right\} .
$$

It follows from the assumptions that the fundamental region of $G_{n-1}$ is compact; i.e., $D_{n-1} / G_{n-1}$ is a compact manifold. The Bergman metric on $D_{n-1}$ projects to the quotient and gives a Hodge metric; therefore $D_{n-1} / G_{n-1}$ is an algebraic variety (cf. Kodaira [3]).

The quotient $U / H_{n-1}=S$ is a compact Riemann surface $\hat{S}$ from which $K_{n-1}$ points $\alpha_{1}, \ldots, \alpha_{K_{n-1}}$ have been removed. Let $\hat{H}_{n-1}$ be a Fuchsian group representing $\hat{S}$ and let $a_{1}, \ldots, a_{K_{n-1}} \in U$ project to $\alpha_{1}, \ldots, \alpha_{K_{n-1}}$ in this representation. Now let $\pi: U \rightarrow U$ be a universal covering map of $U \backslash\left\{g\left(a_{p}\right)\right.$, $\left.1 \leqslant p \leqslant K_{n-1} / g \in \hat{H}_{n-1}\right\}$, that conjugates $H_{n-1}$ onto $\hat{H}_{n-1}$.

For fixed $z \in D_{n-1}$ let $\mu$ denote a Beltrami differential in the class $f_{n-1}(z) \in T\left(H_{n-1}\right)$. We project down to $\hat{H}_{n-1}$ by the formula

$$
\mu \overline{\pi^{\prime}} / \pi^{\prime}=\mu
$$

to obtain a new function $\hat{f}: D_{n-1} \rightarrow T\left(\hat{H}_{n-1}\right)$ associating $z$ to $\hat{\mu}$. We observe that the points $W^{\hat{f}(z)}\left(\alpha_{p}\right), 1 \leqslant p \leqslant k_{n-1}$, are well defined since they are determined entirely by the class $\hat{f}(z)$. Thus we have constructed an extension $\hat{G}$ of $G_{n-1}$ by $\hat{H}_{n-1}$ that acts on a domain $\hat{D}$. Write $M=D / G, \hat{M}=\hat{D} / \hat{G}$. Since $\hat{M}$ is compact there exist functions $\phi_{1}, \ldots, \phi_{n}$ that embed $\hat{M}$ into $\mathbf{P}^{N}$ for some $N$ : the image in $\mathbf{P}^{N}$ is an algebraic variety $A_{0}$.

Finally, the functions

$$
z \mapsto\left(\phi_{1}\left(z, W^{\hat{f}(z)}\left(a_{p}\right)\right), \ldots, \phi_{N}\left(z, W^{\hat{f}(z)}\left(a_{p}\right)\right)\right),
$$

$1 \leqslant p \leqslant K_{n-1}$, induce regular mappings from the algebraic variety $D_{n-1} / G_{n-1}$ into $A_{0}$. The image $B$ is then a Zariski closed set in $A_{0}$ and $M$ is isomorphic to $A_{1}=A_{0} \backslash B$.

\section{REFERENCES}

1. L. Bers, Uniformization, moduli, and Kleinian groups, Bull. London Math. Soc. 4 (1972), 257-300.

2. P. A. Griffiths, Complex analytic properties of certain Zariski open sets on algebraic varieties, Ann. of Math. 94 (1971), 21-51.

3. K. Kodaira and J. Morrow, Complex manifolds, Holt, Rinehart and Winston, Inc., New York, 1971. 
4. G. Riera, Semi-direct products of Fuchsian groups and uniformization, Duke Math. J. 44 (1977), 291-304.

Department of Mathematics, Columbia University, New York, New York 10027

Current address: Matematicas Universidad Tecnica del Estado, Casilla 4823, Correo 2, Santiago, Chile 